\title{
Existence of Fractional Impulsive Functional Integro-Differential Equations in Banach Spaces
}

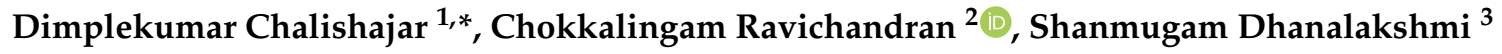 \\ and Rangasamy Murugesu ${ }^{4}$ \\ 1 Department of Applied Mathematics, Virginia Military Institute (VMI), 431, Mallory Hall, Lexington, \\ VA 24450, USA \\ 2 PG and Research Department of Mathematics, Kongunadu Arts and Science College (Autonomous), \\ Coimbatore 641029, Tamil Nadu, India; ravibirthday@gmail.com \\ 3 Department of Mathematics, Kongunadu Arts and Science College (Autonomous), Coimbatore 641029, \\ Tamil Nadu, India; dhana_bala16@yahoo.co.in \\ 4 Department of Mathematics, SRMV College of Arts and Science, Coimbatore 641020, Tamilnadu, India; \\ arjhunmurugesh@gmail.com \\ * Correspondence: chalishajardn@vmi.edu
}

Received: 24 October 2018; Accepted: 27 May 2019; Published: 14 June 2019

\begin{abstract}
In this paper, we establish the existence of piece wise (PC)-mild solutions (defined in Section 2) for non local fractional impulsive functional integro-differential equations with finite delay. The proofs are obtained using techniques of fixed point theorems, semi-group theory and generalized Bellman inequality. In this paper, we used the distributed characteristic operators to define a mild solution of the system. We also discussed the controversy related to the solution operator for the fractional order system using weak and strong Caputo derivatives. Examples are given to illustrate the theory.
\end{abstract}

Keywords: fractional differential equations; impulse; integro-differential equations; non local conditions; fixed point theorem

MSC: 34A08; 34A37; 93B05; 47H10; 45J05

\section{Introduction}

Fractional calculus has gained considerable popularity and importance during the past four decades because fractional derivatives provide an excellent tool for the description of the memory and hereditary properties of various processes. Fractional differential equations produce many applications in many physical phenomena such as seepage flow in porous media and in fluid dynamic traffic models. The most important advantage of using fractional differential equations in these and other applications is their non local property. Also, the study of fractional differential equations has gained considerable importance due to their application in various fields including bio-engineering, mechanics, electrical networks, control theory of dynamical systems, viscoelasticity and so on. Viscoelasticity and related phenomena are of great importance in the study of the mechanical properties of material, especially biological materials. Certain materials show some complex effects in mechanical tests, which cannot be described by a standard linear equation (SLE) mostly owing to shape memory effect during deformation. Recently, researchers have been applying fractional calculus in order to probe the viscoelasticity of such materials with high precision. Fractional calculus is a powerful tool for modeling complex phenomenon. More specifically, modeling of the shape memory phenomena with this powerful tool is studied from different perspectives, as well as presenting physical interpretations. There are different models to evaluate and predict the constitutive equation in the viscoelastic system, 
these models are commonly composed of a different combination of springs and dampers elements. For example, two well-known models are the Maxwell and Kelvin models. The method of distributed characteristic operators is more efficient for defining a mild solution of such systems.

Fractional Calculus is a new powerful tool which has been recently employed to model complex biological systems with non-linear behavior and long-term memory. In recent years, there has been a significant development in fractional differential equations involving fractional derivatives, see the monographs of [1-5] and [6-8]. In Refs. [9-11], authors have studied existence of mild solutions of some semi-linear neutral fractional functional evolution equations with infinite delay with nonlocal conditions. Vijayakumar et al. [12,13] discussed the approximate and exact controllability of fractional neutral integro-differential inclusions with state dependent delay.

The study of impulsive differential equations is linked to their utility in simulating processes and phenomena subject to short time perturbations during their evolution. The perturbations are performed discretely and their duration is negligible in comparison with the total duration of the processes and phenomena. Integro-differential equations play an important role in many branches of linear and non linear functional analysis and their applications in the theory of engineering, mechanics, physics, chemistry, biology, economics, and electrostatics. In recent years, impulsive integro-differential equations have become an important object of investigation stimulated by their numerous applications to problems in mechanics, electrical engineering, medicine, biology, ecology and so forth [14-19]. Gürbüz studied the estimation and behavior of a class of fractional type rough higher order commutators, sublinear operators, and multi-sublinear operators on generalized weighted Morrey spaces [20-25].

The dynamics of many evolutionary processes from various fields such as population dynamics, control theory, physics, biology and medicine undergo abrupt changes at certain moments of time like earth quack, harvesting, shock and so forth. These perturbations can be well approximated as an instantaneous change of states or impulses. These processes are modeled by impulsive differential equations.

The advantage of using non local conditions is that they are measurable at more places and these can be incorporated to get better models. The non local Cauchy problem for the abstract evolution differential equation was first studied by Byszewski [26]. For the importance of non local conditions in different fields, we refer the reader to Refs. $[11,27,28]$.

Recently, in Ref. [29], the authors studied the existence of mild solutions for impulsive fractional semi-linear integro-differential equations using the Banach contraction principle and Schaefer's fixed point theorem. They have considered the system without delay and without non local condition. Our work generalizes the work done in Ref. [29] with abstract formulation. According to our knowledge this is an untreated article in the literature.

Motivated by the above mentioned paper, we study the existence of mild solutions for non local impulsive fractional semi-linear integro-differential equations of the form

$$
\begin{aligned}
{ }^{C} D_{0, t}^{q} x(t)={ }^{C} D_{t}^{q} x(t)=D_{t}^{q} x(t) & =A x(t)+f\left(t, x_{t}, \int_{0}^{t} h\left(t, s, x_{s}\right) d s, \int_{0}^{b} k\left(t, s, x_{s}\right) d s\right), \\
x(0) & =x_{0}+g(x) \in X, \\
\left.\Delta x\right|_{t=t_{k}} & =I_{k}\left(x\left(t_{k}^{-}\right)\right), k=1,2, \cdots, m ; t \in J=[0, b], t \neq t_{k},
\end{aligned}
$$

where ${ }^{C} D_{t}^{q}$ is the Caputo fractional derivative of order $q, 0<q<1$, with lower limit zero, the histories $x_{t}:(-r, 0] \rightarrow X$ are defined by $x_{t}(\theta)=x(t+\theta)$ belongs to a Banach space $X . A: D(A) \subset X \rightarrow X$ is the infinitesimal generator of a strongly continuous semi-group $(T(t))_{t \geq 0}$ of a uniformly bounded operator on $X$, and $A$ is a bounded linear operator. $f: J \times X \times X \times X \rightarrow X$ is jointly continuous, $h, k: J \times J \times X \rightarrow X$ are continuous, $I_{k}: X \rightarrow X$ are impulsive functions, $0=t_{0}<t_{1}<\cdots<t_{m}<$ $t_{m+1}=b,\left.\Delta x\right|_{t=t_{k}}=x\left(t_{k}^{+}\right)-x\left(t_{k}^{-}\right), x\left(t_{k}^{+}\right)=\lim _{h \rightarrow 0^{+}} x\left(t_{k}+h\right)$ and $x\left(t_{k}^{-}\right)=\lim _{h \rightarrow 0^{-}} x\left(t_{k}+h\right)$ represent the right and left limits of $x(t)$ at $t=t_{k}$, respectively. Our method avoids the compactness conditions on 
the semi-group $\{T(t)\}_{t \geq 0}$, and some other hypotheses are more general compared with the previous research papers.

System $\{(1),(2),(3)\}$ described above fits to the mechanical system with impact, the biological phenomenon involving thresholds, the bursting rhythm models and industrial robotics, and many more. In particular, the above mentioned system $\{(1),(2),(3)\}$ and its piecewise (PC)-mild solution (in Section 2) is helpful to generate the tuning and auto-tuning of fractional order controllers for industry applications. Monje et al. [30] design the fractional order $P I^{\lambda} D^{\mu}$ controllers to ensuring a robust performance of the controlled system with respect to gain variations and noise, without using the delay part but can be generalized in a more specific way using the delay for a closed loop and the open loop systems. System $\{(1),(2),(3)\}$ can also be analyzed for a finite time stability test procedure for robotic system where it appears a time delay in fractional control system (refer [31]) but with impulses.

In Section 2, we give some preliminary definitions and lemmas that are to be used later to prove our main results. In Section 3, the existence of $P C$-mild solutions for system $\{(1),(2),(3)\}$ with non-local conditions is discussed. The results are obtained by using the Banach contraction principle and Schaefer's fixed point theorem. An example is given in Section 4 to illustrate the application of our main results.

\section{Preliminaries}

Symbols:

1. $X$ : Banach Space;

2. $P C[J, X]$ : piece-wise continuous function from $[J, X]$;

3. $A(t)$ : infinitesimal generator of a strongly continuous semigroup $T(t)$;

4. $I^{\alpha} f(t)$ : fractional integral of order $\alpha$ for a function $f$;

5. $\quad(R-L) D^{\alpha}$ : Riemann-Liouville (R-L) fractional derivative of order $\alpha>0, n-1<\alpha<n, n \in N$;

6. ${ }^{C} D_{t}^{q}$ : Caputo fractional derivative of order $q, 0<q<1$;

7. ${ }^{C} D_{s}^{\alpha} f(t)$ : strong Caputo derivative of order $\alpha>0$.

Let us consider the set of functions $P C[J, X]=\left\{x: J \rightarrow X \mid x \in C\left[\left(t_{k}, t_{k+1}\right), X\right]\right.$ and there exists $x\left(t_{k}^{+}\right)$and $x\left(t_{k}^{-}\right), k=0,1,2, \cdots, m$ and $\left.x\left(t_{k}^{-}\right)=x\left(t_{k}\right)\right\}$, endowed with the norm $\|x\|_{P C}=\sup _{t \in J}\|x(t)\|$. It is easy to know that $\left(P C[J, X],\|\cdot\|_{P C}\right)$ is a Banach space. Throughout this paper, let $A$ be the infinitesimal generator of a $C_{0}$ semi-group $(T(t))_{t \geq 0}$ of a uniformly bounded operators on $X$ and let $L_{B}(X)$ be the Banach space of all linear and bounded operators on $X$. For a $C_{0}$ semi-group $(T(t))_{t \geq 0}$, we set $M_{1}=\sup _{t \in J}\|T(t)\|_{L_{B}(X)}$.

For each positive constant $r$, set $B_{r}=\{x \in P C[J, X]:\|x\| \leq r\}$.

Let us recall the following known definitions. For more details, see Ref. [32].

Definition 1. The fractional integral of order $\alpha$ with the lower limit zero for a function $f$ is defined as

$$
I^{\alpha} f(t)=\frac{1}{\Gamma(\alpha)} \int_{0}^{t} \frac{f(s)}{(t-s)^{1-\alpha}} d s, \quad t>0, \quad \alpha>0,
$$

provided the right hand-side is point-wise defined on $[0, \infty)$, where $\Gamma(\cdot)$ is the gamma function, which is defined by $\Gamma(\alpha)=\int_{0}^{\infty} t^{\alpha-1} e^{-t} d t$.

Definition 2. The Riemann-Liouville ( $R$-L) fractional derivative of order $\alpha>0, n-1<\alpha<n, n \in N$, is defined as

$$
{ }^{(R-L)} D_{0+}^{\alpha} f(t)=\frac{1}{\Gamma(n-\alpha)}\left(\frac{d}{d t}\right)^{n} \int_{0}^{t}(t-s)^{n-\alpha-1} f(s) d s,
$$


where the function $f(t)$ has absolutely continuous derivative up to order $(n-1)$.

Definition 3. The (strong or classical) Caputo derivative of order $\alpha$ for a function $f \in L^{1}([0, \infty), R)$ given on the interval $[0, \infty)$ is defined by (if it exists)

$$
{ }^{C} D_{s}^{\alpha} f(t)=\frac{1}{\Gamma(n-\alpha)} \int_{0}^{t} \frac{f^{(n)}(s)}{(t-s)^{\alpha+1-n}} d s, \quad t>0, \quad n-1<\alpha \leq n, n \in N .
$$

\section{Remark 1.}

1. R-L and Caputo are just two different operators that are related to each other in a quite simple way. Quite a few details about this are given in the book by Diethelm [2]. There we can also see the exact description of when they are equivalent. The most important difference between them is, of course, the structure of their kernels (i.e., the set of functions that is mapped to zero). Depending on what we want from our operator, one of them or the other one may be the right choice for us. We are using here Caputo because the derivative of a constant is zero for Caputo but not for $R-L$.

2. If a derivative of Caputo type is used instead of $R-L$ type then initial conditions for the corresponding Caputo fractional differential equations can be formulated as for classical ordinary equations, namely $x(0)=x_{0}$.

3. One has to make sure to use a constant function and the Heaviside unit step. They must be considered different so they must have different fractional derivatives. The Heaviside unit step is expected to have a non zero fractional derivative. If one given derivative gives zero it is useless due to the importance it enjoys in practice and its relation with the Dirac delta.

Definition 4. The (weak or generalization of classical) Caputo derivative of order $\alpha$ for a function $f \in$ $L^{1}([0, \infty), R)$ given on the interval $[0, \infty)$ is defined by (if it exists)

$$
{ }^{C} D_{w}^{\alpha} f(t)={ }^{C} D^{\alpha} f(t)=D^{\alpha}\left(f(t)-\sum_{k=0}^{n-1} \frac{t^{k}}{k !} f^{(k)}(0)\right), \quad t>0, \quad n-1<\alpha \leq n, n \in N .
$$

\section{Remark 2.}

1. If $f(t) \in C^{n}[0, \infty)$, then

$$
{ }^{C} D_{w}^{\alpha} f(t)=\frac{1}{\Gamma(n-\alpha)} \int_{0}^{t} \frac{f^{(n)}(s)}{(t-s)^{\alpha+1-n}} d s=I^{n-\alpha} f^{(n)}(t)={ }^{C} D_{s}^{\alpha} f(t), t>0, n-1<\alpha \leq n .
$$

Note that for strong Caputo derivative in Definition $3, f \in C^{n}([0, \infty), R)$ is not necessarily required. In fact, $f^{(n)} \in L^{1}([0, \infty), R)$, for example, $f^{(n-1)}$ be of bounded variation, can guarantee the existence of $D_{s}^{\alpha} f(t)$ on $[0, \infty)$.

2. The Caputo derivative of a constant is equal to zero.

3. If $f$ is an abstract function with values in $X$, then integrals which appear in Definitions 1 and 2 are taken in Bochner's sense. 
Definition 5 ([33]). By a PC-mild solution of the Equations $\{(1),(2),(3)\}$ we mean that a function $x \in P C[J, X]$ which satisfies the following integral equation:

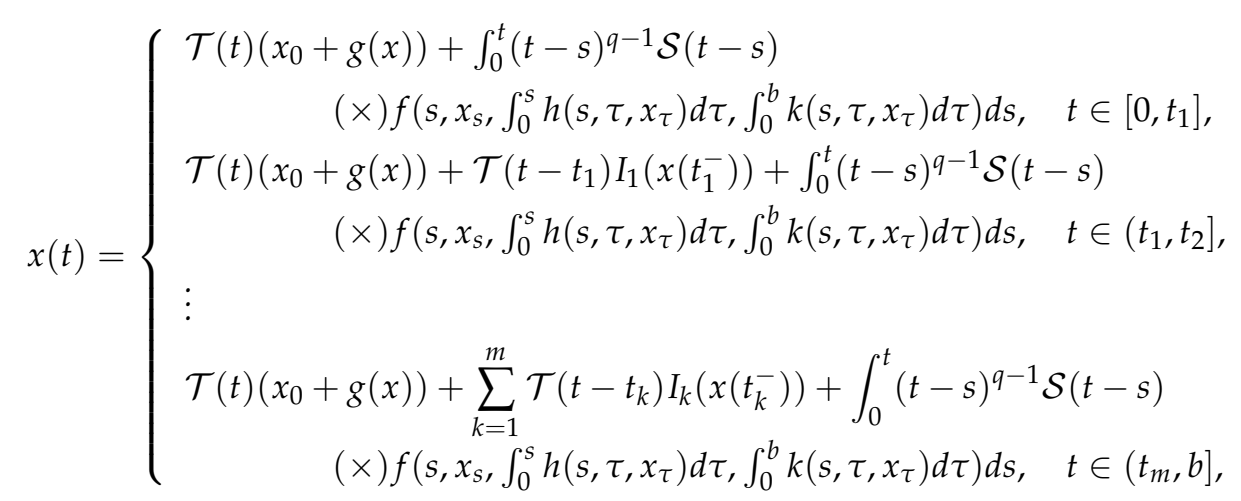

where $\mathcal{T}($.$) and \mathcal{S}($.$) are called characteristic solution operators and given by$

$$
\mathcal{T}(t)=\int_{0}^{\infty} \xi_{q}(\theta) T\left(t^{q} \theta\right) d \theta, \quad \mathcal{S}(t)=q \int_{0}^{\infty} \theta \xi_{q}(\theta) T\left(t^{q} \theta\right) d \theta,
$$

and for $\theta \in(0, \infty)$,

$$
\xi_{q}(\theta)=\frac{1}{q} \theta^{-1-\frac{1}{q}} \omega_{q}\left(\theta^{-\frac{1}{q}}\right) \geq 0, \omega_{q}(\theta)=\frac{1}{\pi} \sum_{n=1}^{\infty}(-1)^{n-1} \theta^{-q n-1} \frac{\Gamma(n q+1)}{n !} \sin (n \pi q),
$$

where $\xi_{q}$ is a probability density function defined on $(0, \infty)$, that is

$$
\xi_{q}(\theta) \geq 0, \quad \theta \in(0, \infty) \text { and } \int_{0}^{\infty} \xi_{q}(\theta) d \theta=1
$$

Remark 3. Controversy on the solution operator, Definition 5, based on Definitions 3 and 4:

1. In this paper, we emphasize that we use the generalized Caputo derivative with the lower bound at zero for Equation (1). However, we have not chosen the classical Caputo derivative and have not changed it in each sub-interval for the Equation (1), where the impulses start at the lower bound $t_{k}$. Obviously, we mean keeping a different one, in each of the impulses the lower bound is at zero. Moreover, Definition 5 is more reasonable since the generalized Caputo derivative in Equation (1) should be fixed at the lower bound at zero once we set the initial time at zero. So we do not expect to change the lower bound again and again in the definition of Caputo derivative for the same equation.

2. We use Definition 4 (generalized Caputo derivative), where the integrable function $f$ can be discontinuous. Definition 4 is more general with respect to Remark 2 (1) (relationship between strong and weak Caputo derivatives). So the result would be wrong if we have used a strong Caputo derivative.

3. Finally, we would like to mention the recently published paper written by Liu and Ahmed [34], where the formula of solutions for semi-linear impulsive fractional Cauchy problems (see (20) in Ref. [34]) coincided with ours (see Definition 5), if one imposes that the semi-linear term and the impulsive term have the same expression in the given interval.

Remark 4. Problems associated with impulsive effects and hereditary properties are modeled by impulsive delay differential equations. So we use impulsive finite delay system $\{(1),(2),(3)\}$ and the solution is a piecewise continuous with discontinuities at impulses time. So, here the mild solution is called PC-mild solution. (As we know a function $x$ is continuous is said to be a mild solution. A function $x$ which is differential almost everywhere on $[0, T]$ is called a strong solution. Clearly, every strong solution is a mild solution, since differentiability implies continuity). 
Definition 6 ([35]). Let $X$ be a Banach space, a one parameter family $T(t), 0 \leq t<+\infty$, of bounded linear operators from $X$ to $X$ is a semi-group of bounded linear operators on $X$ if

1. $T(0)=I$, (here I is the identity operator on $X$ );

2. $T(t+s)=T(t) T(s)$ for every $t, s \geq 0$, (the semi-group property).

A semi-group of bounded linear operator, $T(t)$, is uniformly continuous if $\lim _{t \downarrow 0}\|T(t)-I\|=0$.

Lemma 1 ([35]). Linear operator $A$ is the infinitesimal generator of a uniformly continuous semi-group if and only if $A$ is the bounded linear operator.

Lemma 2 ([36]). (Schaefer's fixed point theorem) Let X be a Banach space and $F: X \rightarrow X$ be a completely continuous operator. If the set

$$
E(F)=\{x \in X: x=\lambda F x \text { for some } 0 \leq \lambda \leq 1\} .
$$

is bounded, then F has at least a fixed point.

Lemma 3 ([33]). The operator $\mathcal{T}(t)$ and $\mathcal{S}(t)$ have the following properties:

1. For any fixed $t \geq 0, \mathcal{T}(t)$ and $\mathcal{S}(t)$ linear and bounded operator, i.e., for any $x \in X$,

$$
\|\mathcal{T}(t) x\| \leq M_{1}\|x\|, \quad\|\mathcal{S}(t) x\| \leq \frac{q M_{1}}{\Gamma(1+q)}\|x\| .
$$

2. $\{\mathcal{T}(t), t \geq 0\}$ and $\{\mathcal{S}(t), t \geq 0\}$ are strongly continuous.

3. $\{\mathcal{T}(t), t \geq 0\}$ and $\{\mathcal{S}(t), t \geq 0\}$ are uniformly continuous, that is, for each fixed $t>0$, and $\epsilon>0$, there exists $h>0$ such that

$$
\begin{array}{r}
\|\mathcal{T}(t+\epsilon)-\mathcal{T}(t)\| \leq \epsilon, \text { for } t+\epsilon \geq 0 \text { and }|\epsilon|<h \\
\|\mathcal{S}(t+\epsilon)-\mathcal{S}(t)\| \leq \epsilon, \text { for } t+\epsilon \geq 0 \text { and }|\epsilon|<h
\end{array}
$$

\section{Existence Results}

In this section, we give the existence of mild solutions of the system $\{(1),(2),(3)\}$. To establish our results, we introduce the following hypotheses:

(H1) $f: J \times X \times X \times X \rightarrow X$ is continuous, and there exist functions $\mu_{1}, \mu_{2}, \mu_{3} \in L\left[J, R^{+}\right]$such that

$$
\begin{array}{r}
\left\|f\left(t, x_{1}, x_{2}, x_{3}\right)-f\left(t, y_{1}, y_{2}, y_{3}\right)\right\| \leq \mu_{1}(t)\left\|x_{1}-y_{1}\right\|+\mu_{2}(t)\left\|x_{2}-y_{2}\right\|+\mu_{3}(t)\left\|x_{3}-y_{3}\right\|, \\
x_{i}, y_{i} \in X, i=1,2,3 .
\end{array}
$$

(H2) $h, k: J \times J \times X \rightarrow X$ is continuous and there exist $M_{h}, M_{k}>0$ such that

$$
\begin{aligned}
\left\|h\left(t, s, x_{1}\right)-h\left(t, s, y_{1}\right)\right\| & \leq M_{h}\left\|x_{1}-y_{1}\right\|, \\
\left\|k\left(t, s, x_{1}\right)-k\left(t, s, y_{1}\right)\right\| & \leq M_{k}\left\|x_{1}-y_{1}\right\|, x_{1}, y_{1} \in X .
\end{aligned}
$$

(H3) $g: P C([0, b], X)$ is continuous and there exists a constant $G>0$ such that

$$
\begin{aligned}
\|g(x)-g(y)\| & \leq G\|x-y\|, \quad \forall x, y \in P C([0, b], X) \\
\|g(0)\| & \leq k_{1} .
\end{aligned}
$$

(H4) The function $I_{k}: X \rightarrow X$ are continuous and there exists $\rho_{k}>0$ such that

$$
\left\|I_{k}(x)-I_{k}(y)\right\| \leq \rho_{k}\|x-y\|, \quad x, y \in X, k=1,2, \cdots, m .
$$


(H5) The function $\Omega_{m}(t): J \rightarrow \mathbb{R}^{+}$is defined by

$$
\Omega_{m}(t)=M_{1}\left(G+m \rho_{m}\right)+\frac{M_{1} b^{q}}{\Gamma(1+q)}\left(\mu_{1}(t)+\mu_{2}(t) M_{h} b+\mu_{3}(t) M_{k} b\right)
$$

where $0<\Omega_{m}(t)<1, t \in J$.

(H6) The constants $\Omega_{u}$ and $\Omega_{m}^{\prime}(t): J \rightarrow \mathbb{R}^{+}$are defined by

$$
\begin{aligned}
\Omega_{u} & =M_{1} K\left(G+m \rho_{m}\right)+\frac{M_{1} b^{q} K}{\Gamma(1+q)}\left(\mu_{1}(t)+\mu_{2}(t) M_{h} b+\mu_{3}(t) M_{k} b\right) \\
\Omega_{m}^{\prime}(t) & =M_{1}\left(G+m \rho_{m}\right)+\frac{M_{1} b^{q}}{\Gamma(1+q)}\left(\mu_{1}(t)+\mu_{2}(t) M_{h} b+\mu_{3}(t) M_{k} b\right)+\frac{M_{1} b^{q} \Omega_{u}}{\Gamma(1+q)}
\end{aligned}
$$

and $0<\Omega_{m}^{\prime}(t)<1, t \in J$.

Theorem 1. If the hypotheses (H1)-(H5) are satisfied, then the nonlocal fractional impulsive integro-differential Equations $\{(1),(2),(3)\}$ has a unique mild solution $x \in P C[J, X]$.

Proof. Define an operator $N$ on $P C[J, X]$ by

$$
(N x)(t)=\left\{\begin{array}{r}
\mathcal{T}(t)\left(x_{0}+g(x)\right)+\int_{0}^{t}(t-s)^{q-1} \mathcal{S}(t-s) \\
\quad f\left(s, x_{s}, \int_{0}^{s} h\left(s, \tau, x_{\tau}\right) d \tau, \int_{0}^{b} k\left(s, \tau, x_{\tau}\right) d \tau\right) d s, \quad t \in\left[0, t_{1}\right] \\
\mathcal{T}(t)\left(x_{0}+g(x)\right)+\mathcal{T}\left(t-t_{1}\right) I_{1}\left(x\left(t_{1}^{-}\right)\right)+\int_{0}^{t}(t-s)^{q-1} \mathcal{S}(t-s) \\
\quad f\left(s, x_{s}, \int_{0}^{s} h\left(s, \tau, x_{\tau}\right) d \tau, \int_{0}^{b} k\left(s, \tau, x_{\tau}\right) d \tau\right) d s, \quad t \in\left(t_{1}, t_{2}\right] \\
\\
\mathcal{T}(t)\left(x_{0}+g(x)\right)+\sum_{k=1}^{m} \mathcal{T}\left(t-t_{k}\right) I_{k}\left(x\left(t_{k}^{-}\right)\right)+\int_{0}^{t}(t-s)^{q-1} \mathcal{S}(t-s) \\
\quad f\left(s, x_{s}, \int_{0}^{s} h\left(s, \tau, x_{\tau}\right) d \tau, \int_{0}^{b} k\left(s, \tau, x_{\tau}\right) d \tau\right) d s, \quad t \in\left(t_{m}, b\right]
\end{array}\right.
$$

We shall show that $N$ is well defined on $P C[J, X]$. For $0 \leq \tau<t \leq t_{1}$, applying (17), we obtain

$$
\begin{aligned}
\|(N x)(t)-(N x)(\tau)\| \leq & \|T(t)-T(\tau)\|\left\|x_{0}+g(x)\right\| \\
& \left\|\int_{0}^{t}(t-s)^{q-1} \mathcal{S}(t-s) f\left(s, x_{s}, \int_{0}^{s} h\left(s, \tau, x_{\tau}\right) d \tau, \int_{0}^{b} k\left(s, \tau, x_{\tau}\right) d \tau\right) d s\right\| \\
\leq & \|T(t)-T(\tau)\|\left[\left\|x_{0}\right\|+G\|x\|+k_{1}\right] \\
& +\left\|\int_{\tau}^{t}(t-s)^{q-1} \mathcal{S}(t-s) f\left(s, x_{s}, \int_{0}^{s} h\left(s, \tau, x_{\tau}\right) d \tau, \int_{0}^{b} k\left(s, \tau, x_{\tau}\right) d \tau\right) d s\right\| \\
& +\| \int_{0}^{\tau}(t-s)^{q-1}[\mathcal{S}(t-s)-\mathcal{S}(\tau-s)] \\
& \quad(\times) f\left(s, x_{s}, \int_{0}^{s} h\left(s, \tau, x_{\tau}\right) d \tau, \int_{0}^{b} k\left(s, \tau, x_{\tau}\right) d \tau\right) d s \| \\
& +\| \int_{0}^{\tau}\left[(t-s)^{q-1}-(\tau-s)^{q-1}\right] \mathcal{S}(\tau-s) \\
& \quad(\times) f\left(s, x_{s}, \int_{0}^{s} h\left(s, \tau, x_{\tau}\right) d \tau, \int_{0}^{b} k\left(s, \tau, x_{\tau}\right) d \tau\right) d s \| .
\end{aligned}
$$

We know that the inequality $\left|t^{\sigma}-\tau^{\sigma}\right| \leq(t-\tau)^{\sigma}$ for $\sigma \in(0,1]$ and $0<\tau \leq t$ and Lemma 3, it is obviously that $\|(N x)(t)-(N x)(\tau)\| \rightarrow 0$ as $t \rightarrow \tau$. Thus, $N x \in\left[\left(0, t_{1}\right], X\right]$. 
For $t_{1}<\tau<t \leq t_{2}$, we have

$$
\begin{aligned}
& \|(N x)(t)-(N x)(\tau)\| \leq \quad \\
& \quad+\mathcal{T}(t)-\mathcal{T}(\tau) \|\left[\left\|x_{0}\right\|+G\|x\|+k\right] \\
& \left.+\left\|\int_{\tau}^{t}\left(t-t_{1}\right)-\mathcal{T}\left(\tau-t_{1}\right)\right\| \|\right)^{q-1} \mathcal{S}(t-s) \\
& \quad(\times) f\left(s, x_{s}, \int_{0}^{s} h\left(s, \tau, x_{\tau}\right) d \tau, \int_{0}^{b} k\left(s, \tau, x_{\tau}\right) d \tau\right) d s \| \\
& +\| \int_{0}^{\tau}(t-s)^{q-1}[\mathcal{S}(t-s)-\mathcal{S}(\tau-s)] \\
& \quad(\times) f\left(s, x_{s}, \int_{0}^{s} h\left(s, \tau, x_{\tau}\right) d \tau, \int_{0}^{b} k\left(s, \tau, x_{\tau}\right) d \tau\right) d s \| \\
& +\| \int_{0}^{\tau}\left[(t-s)^{q-1}-(\tau-s)^{q-1}\right] \mathcal{S}(\tau-s) \\
& \quad(\times) f\left(s, x_{s}, \int_{0}^{s} h\left(s, \tau, x_{\tau}\right) d \tau, \int_{0}^{b} k\left(s, \tau, x_{\tau}\right) d \tau\right) d s \|
\end{aligned}
$$

It is easy to get, as $t \rightarrow \tau$, the right hand side of the above inequality tends to zero. Thus, we can deduce that $N x \in C\left[\left(t_{1}, t_{2}\right], X\right]$. By repeating the same procedure, we can also obtain that $N x \in$ $C\left[\left(t_{2}, t_{3}\right], X\right], \cdots, N x \in C\left[\left(t_{m}, b\right], X\right]$. That is, $N x \in P C[J, X]$. Take $t \in\left(0, t_{1}\right]$, then

$$
\begin{aligned}
\|(N x)(t)-(N y)(t)\| \leq & M_{1} G\|x-y\|_{P C}+\frac{q M_{1}}{\Gamma(1+q)} \int_{0}^{t}(t-s)^{q-1} \\
& {\left[\mu_{1}(s)\left\|x_{s}-y_{s}\right\|+\mu_{2}(s) M_{h} b\left\|x_{s}-y_{s}\right\|+\mu_{3}(s) M_{k} b\left\|x_{s}-y_{s}\right\|\right] d s . }
\end{aligned}
$$

So we deduce that

$$
\|(N x)(t)-(N y)(t)\|_{P C} \leq\left[M_{1} G+\frac{M_{1} b^{q}}{\Gamma(1+q)}\left(\mu_{1}(t)+\mu_{2}(t) M_{h} b+\mu_{3}(t) M_{k} b\right)\right]\|x-y\|_{P C}
$$

For each $t \in\left(t_{1}, t_{2}\right]$, using hypotheses, and (18), we have

$$
\begin{aligned}
\|(N x)(t)-(N y)(t)\|_{P C} \leq & {\left[M_{1}\left(G+\rho_{1}\right)\right.} \\
& \left.+\frac{M_{1} b^{q}}{\Gamma(1+q)}\left(\mu_{1}(t)+\mu_{2}(t) M_{h} b+\mu_{3}(t) M_{k} b\right)\right]\|x-y\|_{P C}
\end{aligned}
$$

In general, for each $t \in\left(t_{i}, t_{i+1}\right]$, using (H5)

$$
\begin{aligned}
\|(N x)(t)-(N y)(t)\|_{P C} \leq & {\left[M_{1}\left(G+m \rho_{m}\right)\right.} \\
& \left.+\frac{M_{1} b^{q}}{\Gamma(1+q)}\left(\mu_{1}(t)+\mu_{2}(t) M_{h} b+\mu_{3}(t) M_{k} b\right)\right]\|x-y\|_{P C} \\
\leq & \Omega_{m}(t)\|x-y\|_{P C} .
\end{aligned}
$$

From the assumption (H5) and in the view of the contraction mapping principle, $N$ has a unique fixed point $x \in P C[J, X]$, that is 


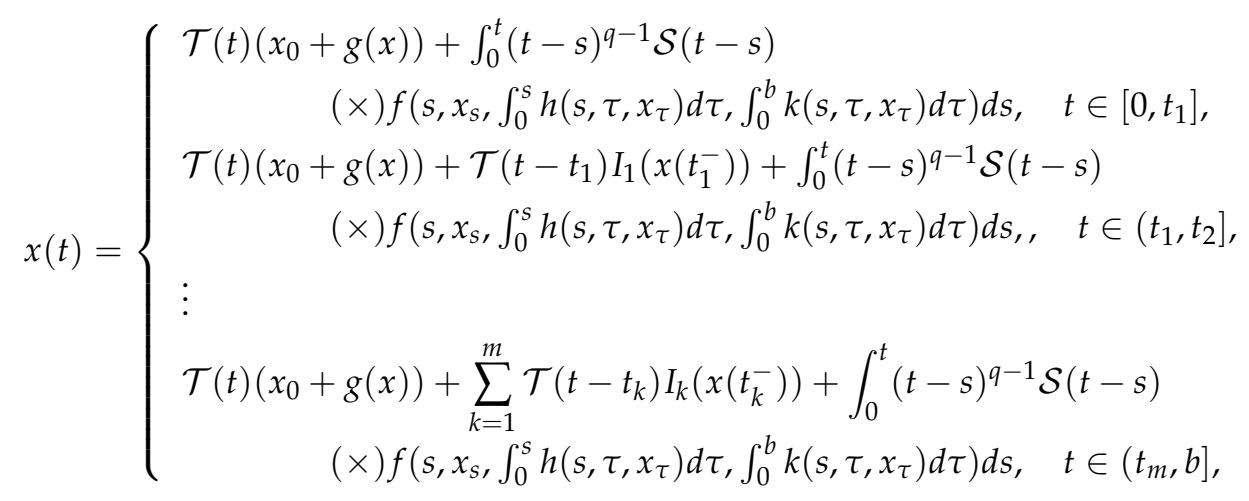

is a PC-mild solution of Equations (1)-(3).

Next theorem is based on Schaefer's fixed point theorem, let us list the following hypotheses:

(H7) $f: J \times X \times X \times X \rightarrow X$ is continuous and there exist functions $c_{1}, c_{2}, c_{3}, c_{4} \in L\left(J, R^{+}\right)$, such that

$$
\|f(t, x, y, z)\| \leq c_{1}(t)+c_{2}(t)\|x\|+c_{3}(t)\|y\|+c_{4}(t)\|z\|, t \in J, x, y, z \in X .
$$

(H8) $h, k: J \times J \times X \rightarrow X$ is continuous and there exist functions $d_{1}, d_{2}, d_{3}, d_{4} \in C\left(I, R^{+}\right)$, such that

$$
\begin{aligned}
& \|h(t, s, x)\| \leq d_{1}(s)+d_{2}(s)\|x\| \\
& \|k(t, s, y)\| \leq d_{3}(s)+d_{4}(s)\|y\|, \quad x, y \in X .
\end{aligned}
$$

(H9) There exist $\Phi_{k} \in C\left[J, R^{+}\right]$, such that

$$
\left\|I_{k}(x)\right\| \leq \Phi_{k}(t)\|x\|, \quad x \in X .
$$

(H10) For all bounded subsets $B_{r}$, the set

$$
\begin{array}{r}
\Pi_{h, \delta}(t)=\left\{\mathcal{T}_{\delta}(t)\left(x_{0}+g(x)\right)+\int_{0}^{t-h}(t-s)^{q-1} \mathcal{S}_{\delta}(t-s) F(s) d s\right. \\
\left.+\sum_{k=1}^{m} \mathcal{T}_{\delta}\left(t-t_{k}\right) I_{k}\left(x\left(t_{k}^{-}\right)\right): x \in B_{r}\right\}
\end{array}
$$

is relatively compact in $X$ for arbitrary $h \in(0, t)$ and $\delta>0$, where

$$
\mathcal{T}_{\delta}(t)=\int_{\delta}^{\infty} \xi_{q}(\theta) \mathcal{T}\left(t^{q} \theta\right) d \theta, \quad \mathcal{S}_{\delta}(t)=q \int_{\delta}^{\infty} \theta \xi_{q}(\theta) \mathcal{T}\left(t^{q} \theta\right) d \theta
$$

(H11) For all bounded subsets $B_{r}$, the set

$$
\begin{array}{r}
\Pi_{h, \delta}^{\prime}(t)=\left\{\mathcal{T}_{\delta}(t)\left(x_{0}+g(x)\right)+\int_{0}^{t-h}(t-s)^{q-1} \mathcal{S}_{\delta}(t-s) F(s) d s\right. \\
\left.+\sum_{k=1}^{m} \mathcal{T}_{\delta}\left(t-t_{k}\right) I_{k}\left(x\left(t_{k}^{-}\right)\right): x \in B_{r}\right\}
\end{array}
$$

is relatively compact in $X$ for arbitrary $h \in(0, t)$ and $\delta>0$.

Theorem 2. If the hypotheses (H6)-(H10) are satisfied, then the non local fractional impulsive integro-differential Equations (1)-(3) has at least one mild solution $x \in P C[J, X]$. 
Proof. From Theorem 1, the operator $N$ is defined as follows:

$$
(N x)(t)=\left\{\begin{array}{c}
\mathcal{T}(t)\left(x_{0}+g(x)\right)+\int_{0}^{t}(t-s)^{q-1} \mathcal{S}(t-s) \\
\quad(\times) f\left(s, x_{s}, \int_{0}^{s} h\left(s, \tau, x_{\tau}\right) d \tau, \int_{0}^{b} k\left(s, \tau, x_{\tau}\right) d \tau\right) d s, \quad t \in\left[0, t_{1}\right], \\
\mathcal{T}(t)\left(x_{0}+g(x)\right)+\mathcal{T}\left(t-t_{1}\right) I_{1}\left(x\left(t_{1}^{-}\right)\right)+\int_{0}^{t}(t-s)^{q-1} \mathcal{S}(t-s) \\
\quad(\times) f\left(s, x_{s}, \int_{0}^{s} h\left(s, \tau, x_{\tau}\right) d \tau, \int_{0}^{b} k\left(s, \tau, x_{\tau}\right) d \tau\right) d s, \quad t \in\left(t_{1}, t_{2}\right], \\
\\
\mathcal{T}(t)\left(x_{0}+g(x)\right)+\sum_{k=1}^{m} \mathcal{T}\left(t-t_{k}\right) I_{k}\left(x\left(t_{k}^{-}\right)\right)+\int_{0}^{t}(t-s)^{q-1} \mathcal{S}(t-s) \\
f\left(s, x_{s}, \int_{0}^{s} h\left(s, \tau, x_{\tau}\right) d \tau, \int_{0}^{b} k\left(s, \tau, x_{\tau}\right) d \tau\right) d s, \quad t \in\left(t_{m}, b\right] .
\end{array}\right.
$$

We shall prove the result in following steps:

Step 1: Continuity of $N$ on $\left(t_{i}, t_{i+1}\right](i=0,1,2, \cdots, m)$

Let $x_{n}, x \in P C[J, X]$ such that $\left\|x_{n}-x^{*}\right\|_{P C} \rightarrow 0(n \rightarrow+\infty)$, then $r=\sup _{n}\left\|x_{n}\right\|_{P C}<\infty$ and $\left\|x^{*}\right\|_{P C}<r$, for every $t \in\left(t_{i}, t_{i+1}\right](i=0,1,2, \cdots, m)$, we have from (19)

$$
\begin{aligned}
\left\|\left(N x_{n}\right)(t)-(N x)(t)\right\| \leq & M_{1} G\left\|x_{n}-x\right\| \\
& +\left\|\sum_{k=1}^{m} T\left(t-t_{k}\right) I_{k}\left(x_{n}\left(t_{k}^{-}\right)\right)-\sum_{k=1}^{m} T\left(t-t_{k}\right) I_{k}\left(x\left(t_{k}^{-}\right)\right)\right\| \\
& +\frac{q M_{1}}{\Gamma(1+q)} \int_{0}^{t}(t-s)^{q-1} \| f\left(s, x_{n_{s}}, \int_{0}^{s} h\left(s, \tau, x_{n_{\tau}}\right) d \tau, \int_{0}^{b} k\left(s, \tau, x_{n_{\tau}}\right) d \tau\right) \\
& -f\left(s, x_{s}, \int_{0}^{s} h\left(s, \tau, x_{\tau}\right) d \tau, \int_{0}^{b} k\left(s, \tau, x_{\tau}\right) d \tau\right) \| d s .
\end{aligned}
$$

Since the functions $f, I_{k}$ and $g$ are continuous,

$$
\begin{aligned}
& f\left(s, x_{n_{s}}, \int_{0}^{s} h\left(s, \tau, x_{n_{\tau}}\right) d \tau, \int_{0}^{b} k\left(s, \tau, x_{n_{\tau}}\right) d \tau\right) \rightarrow \\
& \quad f\left(s, x_{s}, \int_{0}^{s} h\left(s, \tau, x_{\tau}\right) d \tau, \int_{0}^{b} k\left(s, \tau, x_{\tau}\right) d \tau\right), n \rightarrow \infty .
\end{aligned}
$$

By conditions (H7)-(H8) we know that

$$
\begin{array}{r}
\left\|f\left(s, x_{n_{s}} \int_{0}^{s} h\left(s, \tau, x_{n_{\tau}}\right) d \tau, \int_{0}^{b} k\left(s, \tau, x_{n_{\tau}}\right) d \tau\right)-f\left(s, x_{s}, \int_{0}^{s} h\left(s, \tau, x_{\tau}\right) d \tau, \int_{0}^{b} k\left(s, \tau, x_{\tau}\right) d \tau\right)\right\| \\
\leq c_{1}(s)+c_{2}(s)\left\|x_{n_{s}}\right\|+c_{3}(s)\left\|\int_{0}^{s} h\left(s, \tau, x_{n_{\tau}}\right) d \tau\right\|+c_{4}(s)\left\|\int_{0}^{b} k\left(s, \tau, x_{n_{\tau}}\right) d s\right\| \\
+c_{1}(s)+c_{2}(s)\left\|x_{s}\right\|+c_{3}(s)\left\|\int_{0}^{s} h\left(s, \tau, x_{\tau}\right) d \tau\right\|+c_{4}(s)\left\|\int_{0}^{b} k\left(s, \tau, x_{\tau}\right) d s\right\| \\
\leq 2 c_{1}(s)+c_{2}(s)\left(\left\|x_{n}\right\|+\|x\|\right)+2 c_{3}(s) \int_{0}^{s} d_{1}(s)+c_{3}(s) \int_{0}^{s} d_{2}(s)\left(\left\|x_{n}\right\|+\|x\|\right) d s \\
+2 c_{4}(s) \int_{0}^{b} d_{3}(s) d s+c_{4}(s) \int_{0}^{b} d_{4}(s)\left(\left\|x_{n}\right\|+\|x\|\right) d s \\
\leq 2 c_{1}(s)+2 c_{3}(s) \int_{0}^{s} d_{1}(s) d s+2 c_{4}(s) \int_{0}^{b} d_{3}(s) d s \\
+\left(c_{2}(s)+c_{3}(s) \int_{0}^{s} d_{2}(s) d s+c_{4}(s) \int_{0}^{b} d_{4}(s) d s\right)\left(\left\|x_{n}\right\|+\|x\|\right)
\end{array}
$$




$$
\begin{aligned}
\leq 2 c_{1}(s)+2 c_{3}(s) \int_{0}^{s} d_{1}(s) d s+2 c_{4}(s) \int_{0}^{b} d_{3}(s) d s & \\
& +\left(2 c_{2}(s)+2 c_{3}(s) \int_{0}^{s} d_{2}(s) d s+2 c_{4}(s) \int_{0}^{b} d_{4}(s) d s\right) r .
\end{aligned}
$$

Hence,

$$
\begin{aligned}
(t-s)^{q-1} \| f\left(s, x_{n_{s}}, \int_{0}^{s} h\left(s, \tau, x_{n_{\tau}}\right) d \tau, \int_{0}^{b} k\left(s, \tau, x_{n_{\tau}}\right) d \tau\right) & \\
& -f\left(s, x_{s}, \int_{0}^{s} h\left(s, \tau, x_{\tau}\right) d \tau, \int_{0}^{b} k\left(s, \tau, x_{\tau}\right) d \tau\right) \| \in L^{1}\left[J, R^{+}\right] .
\end{aligned}
$$

By the Lebesgue dominated convergence theorem, we get

$$
\begin{aligned}
\int_{0}^{t}(t-s)^{q-1} \| f\left(s, x_{n_{s}}, \int_{0}^{s} h\left(s, \tau, x_{n_{\tau}}\right) d \tau, \int_{0}^{b} k\left(s, \tau, x_{n_{\tau}}\right) d \tau\right) \\
-f\left(s, x_{s}, \int_{0}^{s} h\left(s, \tau, x_{\tau}\right) d \tau, \int_{0}^{b} k\left(s, \tau, x_{\tau}\right) d \tau\right) \| d s \rightarrow 0 .
\end{aligned}
$$

It is easy to get

$$
\lim _{n \rightarrow \infty}\left\|\left(N x_{n}\right)(t)-(N x)(t)\right\|_{P C}=0 .
$$

Thus $N$ is continuous on $\left(t_{i}, t_{i+1}\right],(i=0,1,2, \cdots, m)$.

Step 2: $N$ maps bounded sets into bounded sets in $P C[J, X]$.

From (20) we get

$$
\begin{aligned}
\|(N x)(t)\| \leq & \|\mathcal{T}(t)\|\left\|x_{0}+g(x)\right\|+\frac{q M_{1}}{\Gamma(1+q)} \\
& \quad \int_{0}^{t}(t-s)^{q-1}\left\|f\left(s, x_{s}, \int_{0}^{s} h\left(s, \tau, x_{\tau}\right) d \tau, \int_{0}^{b} k\left(s, \tau, x_{\tau}\right) d \tau\right)\right\| d s \\
& +m\left\|\mathcal{T}\left(t-t_{k}\right) I_{k}\left(x\left(t_{k}^{-}\right)\right)\right\| .
\end{aligned}
$$

and we know that

$$
\begin{gathered}
\left\|f\left(s, x_{s}, \int_{0}^{s} h\left(s, \tau, x_{\tau}\right) d \tau, \int_{0}^{b} k\left(s, \tau, x_{\tau}\right) d \tau\right)\right\| \\
\leq c_{1}(s)+c_{3}(s) \int_{0}^{s} d_{1}(\tau) d \tau+c_{4}(s) \int_{0}^{b} d_{3}(\tau) d \tau \\
\quad+\left(c_{2}(s)+c_{3}(s) \int_{0}^{s} d_{2}(\tau) d \tau+c_{4}(s) \int_{0}^{b} d_{4}(\tau) d \tau\right)\|x\| \\
\leq \Psi_{1}(s)+\Psi_{2}(s)\|x\| .
\end{gathered}
$$

From the above we get,

$$
\begin{aligned}
\|(N x)(t)\| \leq & M_{1}\left(\left\|x_{0}\right\|+G\|x\|+k_{1}\right)+m M_{1} \Phi_{k}\|x\| \\
& +\frac{b^{q} M_{1}}{\Gamma(1+q)} \int_{0}^{t}\left(\Psi_{1}(s)+\Psi_{2}(s)\|x\|\right) d s .
\end{aligned}
$$


Thus for any $x \in B_{r}=\left\{x \in P C[J, X]:\|x\|_{P C} \leq r\right\}$,

$$
\begin{aligned}
\|(N x)(t)\| \leq & M_{1}\left(\left\|x_{0}\right\|+k_{1}\right)+\frac{b^{q} M_{1}}{\Gamma(1+q)} \int_{0}^{b} \Psi_{1}(s) d s \\
& +\left(G+m M_{1} \Phi_{k}+\frac{b^{q} M_{1}}{\Gamma(1+q)} \int_{0}^{t} \Psi_{2}(s) d s\right) r \\
= & \gamma_{1} .
\end{aligned}
$$

Hence $\|(N x)(t)\| \leq \gamma_{1}$, (i.e.,) $N$ maps bounded sets to bounded sets in $P C[J, X]$.

Step 3: $N\left(B_{r}\right)$ is equicontinuous with $B_{r}$ on $\left(t_{i}, t_{i+1}\right](i=0,1,2, \cdots, m)$.

For any $x \in B_{r}, t^{\prime}, t^{\prime \prime} \in\left(t_{i}, t_{i+1}\right](i=0,1,2, \cdots, m)$, we obtain

$$
\begin{aligned}
\left\|(N x)\left(t^{\prime \prime}\right)-(N x)\left(t^{\prime}\right)\right\| \leq & \left\|\mathcal{T}\left(t^{\prime \prime}\right)-\mathcal{T}\left(t^{\prime}\right)\right\|\left\|x_{0}+g(x)\right\| \\
& +\left\|\int_{0}^{t^{\prime \prime}}\left(t^{\prime \prime}-s\right)^{q-1} \mathcal{S}\left(t^{\prime \prime}-s\right) F(s) d s-\int_{0}^{t^{\prime}}\left(t^{\prime}-s\right)^{q-1} \mathcal{S}\left(t^{\prime}-s\right) F(s) d s\right\| \\
& +\left\|\sum_{k=1}^{m} \mathcal{T}\left(t^{\prime \prime}-t_{k}\right) I_{k}\left(x\left(t_{k}^{-}\right)\right)-\sum_{k=1}^{m} T\left(t^{\prime}-t_{k}\right) I_{k}\left(x\left(t_{k}^{-}\right)\right)\right\|
\end{aligned}
$$

after some calculation, we have

$$
\begin{aligned}
\leq & \left\|\mathcal{T}\left(t^{\prime \prime}\right)-\mathcal{T}\left(t^{\prime}\right)\right\|\left\|x_{0}+g(x)\right\|+m\left\|\mathcal{T}\left(t^{\prime \prime}-t^{\prime}\right)\right\|\left\|I_{k}\left(x\left(t_{k}^{-}\right)\right)\right\| \\
& +\left\|\int_{t^{\prime}}^{t^{\prime \prime}}\left(t^{\prime \prime}-s\right)^{q-1} S\left(t^{\prime \prime}-s\right) F(s) d s\right\| \\
& +\left\|\int_{0}^{t^{\prime}}\left[\left(t^{\prime \prime}-s\right)^{q-1}-\left(t^{\prime}-s\right)^{q-1}\right] \mathcal{S}\left(t^{\prime \prime}-s\right) F(s) d s\right\| \\
& +\left\|\int_{0}^{t^{\prime}}\left(t^{\prime}-s\right)^{q-1}\left[\mathcal{S}\left(t^{\prime \prime}-s\right)-\mathcal{S}\left(t^{\prime}-s\right)\right] F(s) d s\right\|
\end{aligned}
$$

Using $\mathcal{T}(t)$ and $\mathcal{S}(t)$ is uniformly continuous and the well known inequality $\left|t^{\prime \sigma}-t^{\prime \prime \sigma}\right| \leq\left(t^{\prime \prime}-t^{\prime}\right)^{\sigma}$ for $\sigma \in(0,1]$ and $0<t^{\prime} \leq t^{\prime \prime}$

$$
\lim _{t^{\prime \prime} \rightarrow t^{\prime}}\left\|(N x)\left(t^{\prime \prime}\right)-(N x)\left(t^{\prime}\right)\right\|=0 .
$$

Thus $N\left(B_{r}\right)$ is equi-continuous with $B_{r}$ on $\left(t_{i}, t_{i+1}\right](i=0,1,2, \cdots, m)$

Step 4: $N$ maps $B_{r}$ into a precompact set in $X$. where

Define $\Pi=N B_{r}$ and $\Pi(t)=\left\{(N x)(t): x \in B_{r}\right\}$ for $t \in J$. Set $\Pi_{h, \delta}(t)=\left\{\left(N_{h, \delta} x\right)(t): x \in B_{r}\right\} ;$

$$
\Pi_{h, \delta}(t)=\left\{\mathcal{T}_{\delta}(t)\left(x_{0}+g(x)\right)+\int_{0}^{t-h}(t-s)^{q-1} S_{\delta}(t-s) F(s) d s+\sum_{k=1}^{m} \mathcal{T}_{\delta}\left(t-t_{k}\right) I_{k}\left(x\left(t_{k}^{-}\right)\right): x \in B_{r}\right\} .
$$

From Lemma 3 (2), (3) and (H10), we can verify that the set $\Pi(t)$ can be arbitrary approximated by the relatively compact set $\Pi_{h, \delta}(t)$. Thus, $N\left(B_{r}\right)(t)$ is relatively compact in $X$. 
Step 5: The set $E=\{x \in P C[J, X]: x=\lambda N x$ for $0<\lambda<1\}$ is bounded.

Let $x \in E$, then

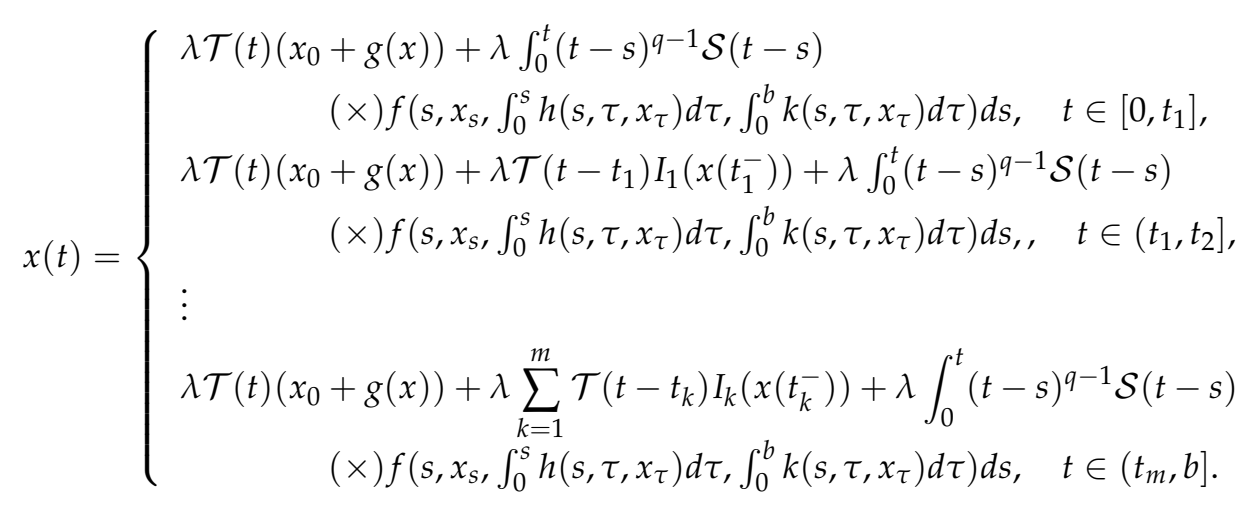

From (30) we know

$$
\begin{aligned}
\|x(t)\| \leq & \lambda M_{1}\left(\left\|x_{0}\right\|+k_{1}\right)+\frac{b^{q} M_{1}}{\Gamma(1+q)} \int_{0}^{b} \Psi_{1}(s) d s \\
& +\lambda\left(G+m M_{1} \Phi_{k}+\frac{b^{q} M_{1}}{\Gamma(1+q)} \int_{0}^{t} \Psi_{2}(s) d s\right)\|x(t)\| .
\end{aligned}
$$

Obviously there exists $\lambda$ sufficiently small such that $\rho=1-M_{1} k_{1} \lambda-\lambda G-\lambda m M_{1} \Phi_{k}>0$ and then we get

$$
\begin{aligned}
\|x(t)\| \leq & \frac{\lambda M_{1}}{\rho}\left\|x_{0}\right\|+\frac{\lambda b^{q} M_{1}}{\rho \Gamma(1+q)} \int_{0}^{b} \Psi_{1}(s) d s \\
& +\frac{\lambda b^{q} M_{1}}{\rho \Gamma(1+q)} \int_{0}^{t} \Psi_{2}(s)\|x(s)\| d s .
\end{aligned}
$$

Let

$$
Q=\lambda M_{1} \rho\left\|x_{0}\right\|+\frac{\lambda b^{q} M_{1}}{\rho \Gamma(1+q)} \int_{0}^{b} \Psi_{1}(s) d s, f(t)=\frac{\lambda b^{q} M_{1}}{\rho \Gamma(1+q)} \int_{0}^{t} \Psi_{2}(s) d s .
$$

It is clear that $f(t)$ is non negative continuous function on $[0,+\infty)$, generalized Bellman inequality implies that

$$
\|x(t)\| \leq Q e^{\int_{0}^{t} f(s) d s} \leq Q e^{\int_{0}^{b} f(s) d s}=C_{0} ;
$$

where $C_{0}$ is a constant. Obviously, the set $E$ is bounded on $\left(t_{i}, t_{i+1}\right],(i=0,1,2, \cdots, m)$. Since $N$ is continuous and compact. From the Schaefer's fixed point theorem, $N$ has a fixed point which is a $P C$-mild solution of $\{(1),(2),(3)\}$. This completes the proof.

\section{Example}

1. Consider the following fractional partial functional mixed differential equations with impulsive conditions of the form 


$$
\begin{aligned}
D_{t}^{q}(z(t, \eta)) & =\frac{\partial}{\partial \eta} z(t, \eta)+\Phi\left(t, z(t, \eta-r), \int_{0}^{t} h_{1}(t, v(x, \eta-r)) d s, \int_{0}^{b} k_{1}(t, v(x, \eta-r)) d s\right), \\
z(t, 0) & =z(t, \pi)=0,0 \leq t \leq T_{0} \quad \text { for }(t, \eta-r) \in\left[0, T_{0}\right] \times(0, \pi), t \neq \frac{T_{0}}{2} \\
z(0, \eta) & =z_{0}(\eta)+g(z(t, \eta)), 0<\eta<\pi \\
\left.\Delta z\right|_{t=\frac{T_{0}}{2}} & =I_{1}\left(\frac{T_{0}^{-}}{2}\right)
\end{aligned}
$$

where $T_{0}>0,0<q<1, D_{t}^{q}$ is a Caputo fractional partial derivative of order $q \in(0,1)$. To write the system (35)-(38) to the form $\{(1),(2),(3)\}$, we take

(i) Let $X=L^{2}([0, \pi])$ as the state space and $z(t, \cdot)=\{z(t, \eta): 0 \leq \eta \leq \pi\}$ as the state;

(ii) $A: D(A) \subset X \rightarrow X$ is defined as $A f=f^{\prime \prime}$ with domain

$$
D(A)=\left\{f \in X: f^{\prime}, f^{\prime \prime} \in X \text { are absolutely continuous, } f(0)=f(\pi)=0\right\} .
$$

Then $A$ is the infinitesimal generator of a strongly continuous semi-group $\{T(t): t \geq 0\}$ in $L^{2}[0, \pi]$. Moreover $T(\cdot)$ is also strongly continuous such that $\|T(t)\| \leq M_{1}$ for each $t \geq 0$. A can be written as $A x=-\sum_{n=1}^{\infty} n^{2}\left(x, e_{n}\right) e_{n}, x \in D(A)$, where $e_{n}(x)=\sqrt{\frac{2}{\pi}} \sin (n x), n \in N$ is an orthonormal set of eigen functions of $A$.

Furthermore, for $x \in X$, we get $T(t) x=\sum_{n=1}^{\infty} \exp \left(-n^{2} t\right)\left(x, e_{n}\right) e_{n}$. We define the operators $f: J \times X \times X \times X \rightarrow X, g: J^{2} \times X \rightarrow X$, and $k: J^{2} \times X \rightarrow X$ by

$$
x_{t}=z(t, n-r), h\left(t, s, x_{s}\right)=h_{1}(t, v(x, n-r)), k\left(t, s, x_{s}\right)=k_{1}(t, v(x, n-r)) .
$$

Obviously, $h_{1}, k_{1}$ satisfy Lipschitz condition (H2), $g$ satisfies Lipschitz condition (H3), and $I_{1}$ satisfies Lipschitz condition (H4). All together satisfy (H1). Also it is easy to verify conditions (H5) and (H6).

Thus functions $\Phi, h_{1}, k_{1}, g$ and $I_{1}$ of the system (35)-(38) satisfies the hypotheses of the Theorems 1 and 2. Thus all the conditions of Theorems 1 and 2 are satisfied. Therefore the system (35)-(38) can be written to the abstract form $\{(1),(2),(3)\}$. That phenomenon models Equations (35)-(38). Hence, we conclude that the system (35)-(38) has a mild solution.

2. Consider the following numerical fractional partial functional differential equations with impulsive conditions of the form

$$
\begin{gathered}
{ }^{C} D^{\frac{3}{2}} x(t)=\frac{1}{(t+63)} \frac{|x(t)|}{1+|x(t)|}+\frac{e^{-t}}{\left(62+e^{t}\right)} \frac{|x(t / 2)|}{1+|x(t / 2)|}+\frac{1}{63} \int_{0}^{t}\left(\frac{e^{-s}}{7}\right) \frac{\left|x\left(s^{2}\right)\right|}{1+\left|x\left(s^{2}\right)\right|} d s, \quad t \in[0,1], \\
\left.\Delta x\right|_{t=t_{k}}=I_{k}\left(x\left(t_{k}^{-}\right)\right), k=1,2, \cdots ; x(0)=x_{0}+g(x) .
\end{gathered}
$$

Above system can be written as an abstract formulation of system $\{(1),(2),(3)\}$.

\section{Conclusions}

Here, we have established the existence of PC-mild solutions for non local fractional impulsive functional integro-differential equations with finite delay. The proofs are obtained using the Banach contraction principle and a fixed point theorem due to Schaefer with generalized Bellman inequality. We have used the distributed characteristic operators to define the mild solution of the system. We took care of all the controversy related to the solution operator (refer to Remark 3), which has not been 
discussed in the literature. Distributed characteristics for fractional systems are a powerful tool for modeling complex phenomena. More specifically, modeling of the shape memory phenomena with this powerful tool is studied from different perspectives, as well as presenting some physical interpretation. Using the proposed method, we can evaluate different models and predict the constitutive equation in the viscoelastic system; these models are commonly composed of a different combination of springs and dampers elements.

We have also considered a bounded linear operator which gave the standard semi-group in the exponential form, but one can consider an unbounded operator and prove the results in future work. For this we refer to the techniques given in Refs. [37,38]. The same problem can also be extended for the Trajectory controllability problem, which is a new direction to the field of fractional order differential and partial differential equations with its numerical approach, refer to Ref. [39]. It is always interesting to verify analytical and numerical results.

System (1) can be extended to couple systems of nonlinear fractional equations with slit-strips-type integral boundary condition (we refer to Ref. [40] and references cited therein), which may enhance a new direction in the field of fractional order systems.

Author Contributions: All authors have made the same contribution. All authors read and approved the final manuscript.

Funding: This research received no external funding.

Acknowledgments: The authors wish to thank the anonymous reviewers for their valuable comments and suggestions.

Conflicts of Interest: The authors declare no conflicts of interest.

\section{References}

1. Abbas, S.; Benchohra, M.; N'Guérékata, G.M. Topics in Fractional Differential Equations; Springer: New York, NY, USA, 2012.

2. Diethelm, K. The Analysis of Fractional Differential Equations; Springer: Berlin, Germany, 2010.

3. Podlubny, I. Fractional Differential Equations; Academic Press: New York, NY, USA, 1999.

4. Tarasov, V.E. Fractional Dynamics, Application of Fractional Calculus to Dynamics of Particles; Fields and Media; Springer: Berlin, Germany, 2010.

5. Miller, K.S.; Ross, B. An Introduction to the Fractional Calculus and Fractional Differential Equations; Wiley: New York, NY, USA, 1993.

6. Chen, F.; Chen, A.; Wang, X. On solutions for impulsive fractional functional differential equations. Differ. Equ. Dyn. Syst. 2009, 17, 379-391. [CrossRef]

7. Darwish, M.A.; Henderson, J.; Ntouyas, S.K. Fractional order semi-linear mixed type functional differential equations and inclusions. Nonlinear Stud. 2009, 16, 197-219.

8. Machado, J.A.; Ravichandran, C.; Rivero, M.; Trujillo, J.J. Controllability results for impulsive mixed type functional integro-differential evolution equations with non-local conditions. Fixed Point Theory Appl. 2013, 66, 1-16.

9. Mophou, G.M.; N'Guérékata, G.M. Mild solutions for semi-linear fractional differential equations. Electron. J. Differ. Equ. 2009, 2009, 1-9.

10. Mophou, G.M.; N'Guérékata, G.M. Existence of mild solutions of some semi-linear neutral fractional functional evolution equations with infinite delay. Appl. Math. Comput. 2010, 216, 61-69.

11. Mophou, G.M.; N'Guérékata, G.M. Existence of mild solution for some fractional differential equations with nonlocal conditions. Semigroup Forum 2009, 79, 315-322. [CrossRef]

12. Vijayakumar, V.; Ravichandran, C.; Murugesu, R. Existence of mild solutions for nonlocal Cauchy problem for fractional neutral evolution equations with infinite infinite delay. Surv. Math. Appl. 2014, 9, 117-129.

13. Vijayakumar, V.; Ravichandran, C.; Murugesu, R. Approximate controllability for a class of fractional neutral integro-differential inclusions with state dependent delay. Nonlinear Stud. 2013, 20, 511-530.

14. Aissani, K.; Benchohra, M. Fractional integro-differential equations with state dependent delay. Adv. Dyn. Syst. Appl. 2014, 9, 17-30. 
15. Chalishajar, D.N.; Karthikeyan, K.; Anguraj, A. Existence results for impulsive perturbed partial neutral functional differential equations in Frechet spaces. Dyn. Contin. Discret. Impuls. Syst. Ser. A Math. Anal. 2015, 22, 25-45.

16. Chalishajar, D.N.; Karthikeyan, K.; Trujillo, J.J. Existence of Mild Solutions for Fractional Impulsive Semi-linear Integro-differential Equations in Banach Spaces. Commun. Appl. Nonlinear Anal. 2012, 19, 45-56.

17. Debbouche, A.; Baleanu, D. Controllability of fractional evolution non-local impulsive quasilinear delay integro-differential systems. Comput. Math. Appl. 2011, 62, 1442-1450. [CrossRef]

18. Hernández, E.; Rabello, M.; Henriquez, H. Existence of solutions for impulsive partial neutral functional differential equations. J. Math. Anal. Appl. 2007, 331, 1135-1158. [CrossRef]

19. Shu, X.B.; Lai, Y.; Chen, Y. The existence of mild solutions for impulsive fractional partial differential equations. Nonlinear Anal. 2011, 74, 2003-2011. [CrossRef]

20. Pandey, D.N.; Ujlayan, A.; Bahuguna, D. On solution to fractional order integro-differential equations with analytic semi-groups. Nonlinear Anal. 2009, 71, 3690-3698. [CrossRef]

21. Gürbüz, F. On the behavior of a class of fractional type rough higher order commutators on generalized weighted Morrey spaces. J. Coupled Syst. Multiscale Dyn. 2018, 6, 191-198. [CrossRef]

22. Gürbüz, F. Sublinear operators with rough kernel generated by fractional integrals and their commutators on generalized Morrey spaces. J. Sci. Eng. Res. 2017, 4, 144-163.

23. Gürbüz, F. Some estimates for generalized commutators of rough fractional maximal and integral operators on generalized weighted Morrey spaces. Canad. Math. Bull. 2017, 60, 131-145. [CrossRef]

24. Gürbüz, F. Sublinear operators with a rough kernel generated by fractional integrals and local Campanato space estimates for commutators with rough kernel on generalized local Morrey spaces. Int. J. Appl. Math. Stat. (IJAMAS) 2017, 56, 52-62.

25. Gürbüz, F. Multi-sublinear operators generated by multilinear fractional integral operators and local Campanato space estimates for commutators on the product generalized local Morrey spaces. Adv. Math. (China) 2018, 47, 855-880.

26. Byszewski, L. Theorems about the existence and uniqueness of solutions of semi-linear evolution nonlocal Cauchy problem. J. Math. Anal. Appl. 1991, 162, 494-505. [CrossRef]

27. Byszewski, L. Existence of solutions of semi-linear functional differential evolution non-local problem. Nonlinear Anal. 1998, 34, 65-72. [CrossRef]

28. Zhang, X.; Huang, X.; Liu, Z. The existence and uniqueness of mild solutions for impulsive fractional equations with nonlocal conditions and infinite delay. Nonlinear Anal. Hybrid Syst. 2010, 4, 775-781. [CrossRef]

29. Qin, H. ; Zuo, X.; Liu, J. Existence and Controllability results for fractional impulsive integrodifferential systems in Banach Spaces. Abstr. Appl. Anal. 2013, 2013, 295837. [CrossRef]

30. Monje, C.A.; Vinagre, B.M.; Feliu, V.; Chen, Y. Tuning and auto-tuning of fractional order controllers for industry applications. Control Eng. Pract. 2008, 16, 798-812. [CrossRef]

31. Lazarevic, M.P. Finite time stability analysis of PD fractional control of robotic time-delay systems. Mech. Res. Commun. 2006, 33, 269-279. [CrossRef]

32. Baleanu, D.; Diethelm, K.; Scalas, J.E.; Trujillo, J. Fractional Calculus Models and Numerical Methods; Series on Complexity, Nonlinearity and Chaos; World Scientific: Singapore, 2012.

33. Wang, J.R.; Feckan, M.; Zhou, Y. On the new concept of solutions and existence results for impulsive fractional evolution equations. Dyn. Part. Differ. Equ. 2011, 8, 345-361.

34. Liu, Y.; Ahmad, B. A study of impulsive multiterm fractional differential equations with single and multiple base points and applications. Sci. World J. 2014, 2014, 194346. [CrossRef]

35. Pazy, A. Semi-Groups of Linear Operators and Applications to Partial Differential Equations; Applied Mathematical Sciences; Springer, New York, NY, USA, 1983; Volume 44.

36. Schaefer, H. Uber die methode der a priori schranken. Math. Annal. 1955, 129, 415-416.

37. Chalishajar, D.N.; Acharya, F.S. Controllability of Neutral Impulsive Differential Inclusions with Non-Local Conditions. Appl. Math. 2011, 2, 1486-1496. [CrossRef]

38. Chalishajar, D.N.; Heena Chalishajar, F.S. Acharya, Controllability of Second Order Neutral Impulsive Differential Inclusions with non-local conditions. Dyn. Contin. Discret. Impuls. Syst. Ser. A Math. Anal. 2012, 19, 107-134. 
39. Chalishajar, D.N.; Heena Chalishajar, F.S. Trajectory Controllability of Second Order Nonlinear Integro-Differential System: An Analytical and a Numerical Estimation. In Differential Equations and Dynamical System; Springer: Berlin, Germany, 2014. [CrossRef]

40. Mahmudov, N.I.; Al Khateeb, A. Stability, existence and uniqueness of a coupled system of fractional differential equations with integral boundary condditions. Mathematics 2019, 7, 354. [CrossRef]

(C) 2019 by the authors. Licensee MDPI, Basel, Switzerland. This article is an open access article distributed under the terms and conditions of the Creative Commons Attribution (CC BY) license (http:/ / creativecommons.org/licenses/by/4.0/). 